\title{
Linear One-sided Stability of MAT for Weakly Injective 3D Domain
}

\author{
Sung Woo Choi \\ Max Planck Institute for Computer Science \\ Stuhlsatzenhausweg 85 \\ D-66123, Saarbrücken, Germany \\ swchoi@mpi-sb.mpg.de
}

\author{
Hans-Peter Seidel \\ Max Planck Institute for Computer Science \\ Stuhlsatzenhausweg 85 \\ D-66123, Saarbrücken, Germany \\ hpseidel@mpi-sb.mpg.de
}

\begin{abstract}
Despite its usefulness in many applications, the medial axis transform (MAT) is very sensitive to the change of the boundary in the sense that, even if a shape is perturbed only slightly, the Hausdorff distance between the MATs of the original shape and the perturbed one may be large. However, it is known that MATs of 2D domains are stable if we view this phenomenon with the one-sided Hausdorff distance. This result depends on the fact that MATs are stable if the differences between them are measured with the recently introduced hyperbolic Hausdorff distance. In this paper, we extend the result for the one-sided stability of the MAT to a class of 3D domains called weakly injective, which contains many important 3D shapes typically appearing in solid modeling. Especially, the weakly injective 3D domains can have sharp features like corners or edges. In fact, by using the stability of the MAT under the hyperbolic Hausdorff distance, we obtain an explicit bound for the one-sided Hausdorff distance of the MAT of a weakly injective 3D domain with respect to that of a perturbed domain, which is linear with respect to the domain perturbation. We discuss some consequences of this result concerning the computation and the approximation of the medial axis transform of 3D objects.
\end{abstract}

\section{Categories and Subject Descriptors}

I.3.5 [Computer Graphics]: Computational Geometry and Object Modeling-curve, surface, solid, and object representations, hierarchy and geometric transformations; G.1.2 [Numerical Analysis]: Approximation-approximation of surfaces and contours; I.5.1 [Pattern Recognition]: Models-geometric; I.4.10 [Image Processing and Computer Vision]: Image Representation-volumetric, morphological; I.4.7 [Image Processing and Computer Vision]: Feature Measurement—feature representation

\section{General Terms}

Theory, Reliability, Measurement, Algorithm

Permission to make digital or hard copies of all or part of this work for personal or classroom use is granted without fee provided that copies are not made or distributed for profit or commercial advantage and that copies bear this notice and the full citation on the first page. To copy otherwise, to republish, to post on servers or to redistribute to lists, requires prior specific permission and/or a fee.

SM'02, June 17-21, 2002, Saarbrucken, Germany.

Copyright 2002 ACM 1-58113-506-8/02/0006 ...\$5.00.

\section{Keywords}

medial axis transform, skeleton, stability, Hausdorff distance, hyperbolic Hausdorff distance, weakly injective domain

\section{INTRODUCTION}

For a given a shape in a Euclidean space, its medial axis (MA) is defined as the set of the centers of maximal inscribed balls contained in the shape, and the medial axis transform (MAT) is defined as the set of all the pairs of medial axis point and the radius of the corresponding inscribed ball. While the MA captures the overall features of a shape in a compact form, MAT has the full information about a shape, which is enough to reconstruct it. More precisely, the medial axis transform $\mathbf{M A T}(\Omega)$ and the medial axis $\mathbf{M A}(\Omega)$ of an $n$-dimensional domain $\Omega$ is defined by

$$
\begin{aligned}
& \operatorname{MAT}(\Omega)=\left\{(p, r) \in \mathbb{R}^{n} \times[0, \infty) \mid\right. \\
& \left.B_{r}(p) \text { is a maximal ball contained in } \Omega\right\}, \\
& \mathbf{M A}(\Omega)=\left\{p \in \mathbb{R}^{n} \mid \exists r \geq 0, \text { s.t. }(p, r) \in \mathbf{M A T}(\Omega)\right\} .
\end{aligned}
$$

Since its introduction [3], MAT has been one of the most intensively investigated and widely used objects in shape analysis. Some of its applications include: biological shape recognition [17], character recognition and representation [21], fingerprint classification [14], and visual analysis of circuit boards [22]. In general, it shrinks the dimension of a given shape by 1 , while preserving the the topology in a homotopically equivalent way $[3,4,20]$. It also has a natural definition closely connected to the Voronoi diagram, which can in fact be utilized in some applications [1].

The analysis of the MAT in 2D concerns mostly about dealing with the images, and hence has a large impact in fields like image processing, pattern recognition, and computer vision. On the other hand, 3D MAT is becoming more important in fields such as CAD, computer graphics, and mechanical engineering, which should naturally deal with $3 \mathrm{D}$ objects. But compared to the vast amount of works on the MAT in 2D, the corresponding works in 3D are still in a premature state in view of their importance. This is in part caused by the fact that there are few existing theoretical bases for the 3D MAT. In fact, the exact geometry of the 3D MAT has not been understood completely yet $[12,13,19,20]$. Thus basic theoretical analysis on the geometry of the 3D MAT is much in need at present.

In the meanwhile, one nuisance in using the MAT is its notorious instability: In general, MAT is not stable under the perturbation of domains [2, 16]. See Figure 1: Even when the upper domain is slightly perturbed to the lower domain, their corresponding MATs (MAs) have a drastic difference. 

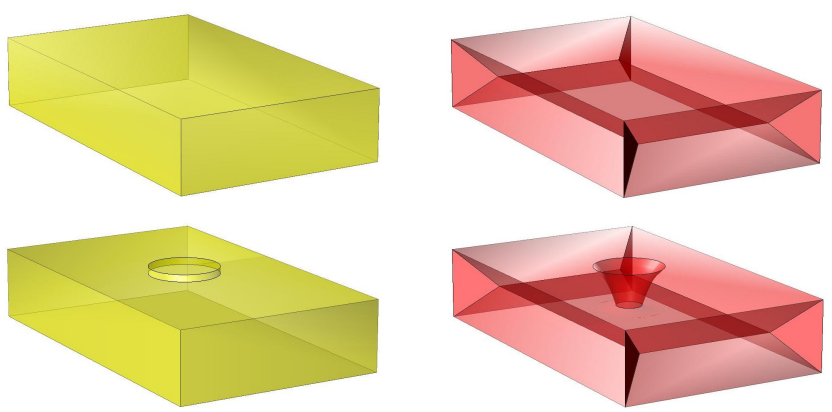

Figure 1: Instability of MAT: A small perturbation (under the Hausdorff distance) of the domain on the upper left to the one on the lower left leads to a drastic change in their corresponding MATs (upper right and lower right, resp.). But the onesided Hausdorff distance of the original MAT (upper right) with respect to the perturbed one (lower right) still remains small.

Combined with the well-known difficulty of computing the medial axis transform, this phenomenon can cause a lot of problems in real applications, since, in many cases, shapes in problems come with inevitable noises. In $2 \mathrm{D}$, there have been many attempts to overcome this difficulty by reducing the complexity of the MAT by "pruning" out the less important parts $[10,15,18]$. Also in 3D, there have been attempts to approximate a more stable part of the MAT rather than to compute the whole complex MAT [1]. But most of these attempts come without precise error analysis, thus lacking in firm theoretical guarantee (with some notable exceptions including [1]).

While the instability of the MAT is inherent from its definition, we still can have one important observation from Figure 1; The original MAT (upper right) is contained approximately in the perturbed MAT (lower right). More precisely, the one-sided Hausdorff distance of the original MAT with respect to the perturbed one still changes little. In this paper, we will closely analyze this phenomenon, and show that this is in fact a general property of the MAT regarding its stability. In particular, for a class of the $3 \mathrm{D}$ domains called weakly injective, we will show that the onesided Hausdorff distance of a given original MAT with respect to a perturbed one can be bounded linearly with the magnitude of the perturbation on the domain.

Weakly injective domains are domains without degenerate types of end points in their MATs so that they satisfy some smoothness condition. They include many domains that naturally arise in solid modeling and CAD applications, and, especially, they can still have sharp corners or edges. In fact, the weakly injective domains form the largest class of 3D domains with linear bound for the one-sided Hausdorff distance of the MAT, and the constant for this linear bound can serve as a new indicator of the level of detail for a given 3D shape.

These results generalize the corresponding ones in 2D [7], which will be reviewed in Section 4. We mention that the proofs given here for the $3 \mathrm{D}$ case can obviously be generalized to any higher dimension. As well as the theoretical importance of these results, we will also point out some possible effects they will have on the general computation of the 3D MAT.

\section{GEOMETRY OF MAT}

In this section, we explore some facts about the geometry of the MAT in 2D and 3D, which are relevant to our further analysis.

\subsection{Situation in 2D}

For 2D domains, much about the geometry of the MAT are known. The most basic and fundamental fact is the following:

PROPOSITION 1. [4]

Let $\Omega$ be a normal domain in $2 D$. Then $\mathbf{M A}(\Omega)$ and $\operatorname{MAT}(\Omega)$ have finite graph structures, and $\mathrm{MA}(\Omega)$ is homotopically equivalent to $\Omega$.

Here, the condition 'normal' is important. We call a compact 2D domain $\Omega$ normal, if its boundary $\partial \Omega$ consists of finitely many simple closed curves, each of which in turn consists of finitely many real-analytic curve pieces. Though the real-analycity seems rather strong, $\operatorname{MAT}(\Omega)$ and $\mathbf{M A}(\Omega)$ may not be graphs with finite structure, unless the original domain $\Omega$ satisfies the normality condition [4]. Furthermore, most domains in applications fall into the category of normal domains. So it is natural to consider only the normal domains.

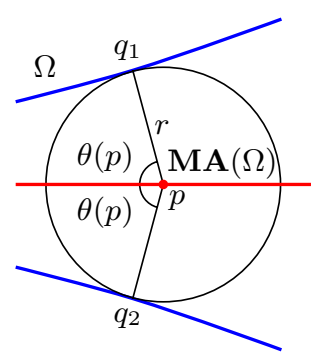

(a)

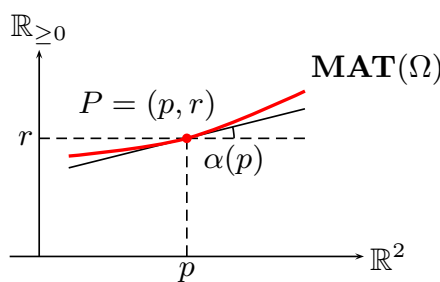

(b)
Figure 2: Local geometry of 2D MA and MAT around a generic point: MA and MAT are $C^{1}$ curves around a generic point.

Normal domains also have the following nice properties: Let $\Omega$ be a normal domain. Then except for some finite number of special points, the maximal ball $B_{r}(p)$ for every $P=(p, r) \in \operatorname{MAT}(\Omega)$ has exactly two contact points with the boundary $\partial \Omega$. See Figure 2 . Around such $p$ and $P, \mathbf{M A}(\Omega)$ and $\mathbf{M A T}(\Omega)$ are $C^{1}$ curves in $\mathbb{R}^{2}$ and in $\mathbb{R}^{2} \times \mathbb{R}_{\geq 0}$ respectively. Here, we denote $\mathbb{R}_{\geq 0}=\{x \in$ $\mathbb{R} \mid x \geq 0\}$. For every $p$ in the set of such generic points in $\mathbf{M A}(\Omega)$ (denoted by $G(\Omega)$ ), we define the angle $0<\theta(p) \leq \frac{\pi}{2}$ to be the angle between $\overline{p q_{1}}$ (or equivalently $\overline{p q_{2}}$ ) and $\mathbf{M A}(\Omega)$ at $p$, where $q_{1}, q_{2}$ are the two contact points (See Figure 2 (a)). We also define the angle $0 \leq \alpha(p)<\frac{\pi}{2}$ to be the angle which $\operatorname{MAT}(\Omega)$ makes with the point plane $\mathbb{R}^{2}$ at $P$ (See Figure $2(b)$ ). Then it is easy to see the following general relation:

$$
\cos \theta(p)=\tan \alpha(p),
$$

for every $p \in G(\Omega)$.

Now, for every normal domain $\Omega$ (except for the special case of circular disks), we define

$$
\begin{aligned}
\theta_{\Omega} & =\inf \{\theta(p): p \in G(\Omega)\}, \\
\alpha_{\Omega} & =\sup \{\alpha(p): p \in G(\Omega)\} .
\end{aligned}
$$

Note that $0 \leq \theta_{\Omega} \leq \frac{\pi}{2}, 0 \leq \alpha_{\Omega} \leq \frac{\pi}{4}$, and, from (1), we have $\cos \theta_{\Omega}=\tan \alpha_{\Omega}$. When $\Omega$ is a circular disk, we define $\theta_{\Omega}=\frac{\pi}{2}$ and $\alpha_{\Omega}=0$. We also define $\rho_{\Omega}=\min \{r:(p, r) \in \operatorname{MAT}(\Omega)\}$, which is the smallest radius of the maximal balls contained in $\Omega$. 


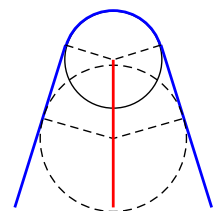

(a)

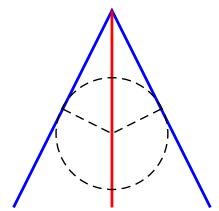

(b)

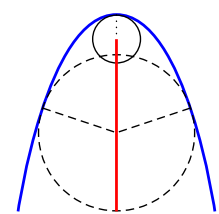

(c)
Figure 3: Three types of 1-prong points for 2D MAT

We call an end point of MA a 1-prong point. There are exactly three types of 1-prong points in MA. See Figure 3: Type (a) is a 1-prong point with a contact arc, Type (b) is a sharp corner, and Type (c) is a degenerate case when it is the center of a maximal circle with only one contact point at which the circle osculates the boundary. Especially, the center of a circular disk is a 1-prong point of Type (a). It is easy to see that $\theta_{\Omega}=0$, if and only if $\mathbf{M A}(\Omega)$ has a 1-prong point of Type (c), and $\rho_{\Omega}=0$, if and only if $\mathbf{M A}(\Omega)$ has a 1-prong point of Type (b).

We call a normal domain $\Omega$ injective, if $\theta_{\Omega}>0$ and $\rho_{\Omega}>0$, and weakly injective, if $\theta_{\Omega}>0$. Thus, $\Omega$ is injective, if and only if every 1-prong point of $\mathbf{M A}(\Omega)$ is of Type (a), and it is weakly injective, if and only if $\mathbf{M A}(\Omega)$ does not have a 1-prong point of Type (c). Note that a weakly injective domain may have a sharp corner (i.e., Type (b)), while an injective domain may not.

\subsection{Situation in 3D}
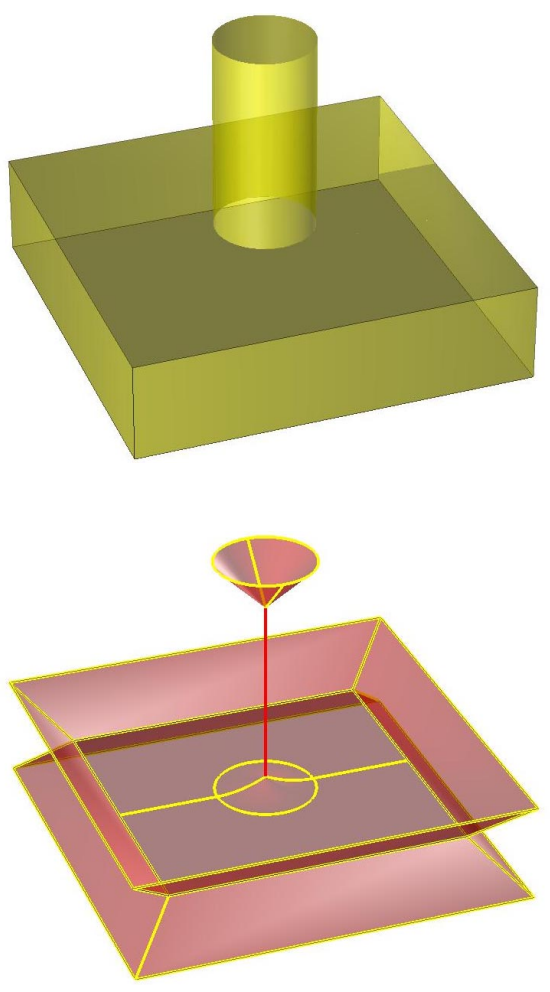

Figure 4: MA (MAT) can be decomposed into finitely many patches called elements. Each patch has trivial topology, and according to its dimension, it is either called a surface element or a line element. Note that such a decomposition is not unique.

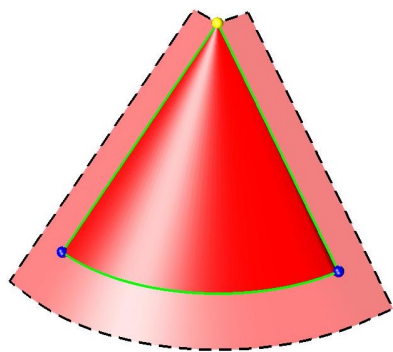

(a)

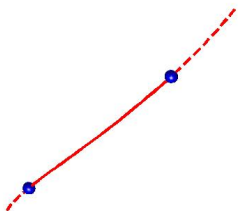

(b)
Figure 5: Two types of the elements in MA: (a) A surface element (left), and (b) a line element (right). Both can be extended slightly at their boundaries in the $C^{1}$ manner (so that the resulting extensions are locally $C^{1}$ manifolds at the boundaries), with possible exceptions at some boundary points of a surface element (c.f., the top corner point in (a)).

Compared to the 2D case, the geometry of the 3D MAT is not well understood yet. In fact, there still does not exist a result in 3D which is analogous to Proposition 1. In general, MAs (resp., MATs) of 3D domains would be (2-dimensional) $\mathrm{CW}$-complexes in $\mathbb{R}^{3}$ (resp., $\mathbb{R}^{3} \times \mathbb{R}_{\geq 0}$ ), which means that they can be decomposed into simpler 2D and 1D patches. See Figure 4 for an example of such decomposition. But, of course, they are not the most general types of CW-complexes, since they are MAs and MATs of specific 3D domains. In view of the 2D situation, we introduce the following reasonable conditions for MA and MAT in our analysis:

1. They can be decomposed into a finite number of parts called elements.

2. An element has one of the following two forms:

(a) Surface Element (Figure 5 (a)): A 2-dimensional $C^{1}$ manifold with boundary, embedded in $\mathbb{R}^{3}$ (or $\mathbb{R}^{3} \times$ $\mathbb{R}_{\geq 0}$ ), which is homeomorphic to a closed disk, and whose boundary consists of finitely many $C^{1}$ curve pieces.

(b) Line Element (Figure 5 (b)): A $C^{1}$ curve piece (without self intersections) embedded in $\mathbb{R}^{3}$ (or $\mathbb{R}^{3} \times \mathbb{R}_{\geq 0}$ ) including its two end points.

3. Each pair of two different elements can meet only at their boundaries.

4. If a MAT point $P=(p, r)$ is in the interior of a surface element, then the maximal ball $B_{r}(p)$ has exactly two contact points with the domain boundary. If $P$ is in the interior of a line element, the contact points form a circle.

5. A line element can be extended slightly at their boundaries in the $C^{1}$ manner (See Figure 5 (b)). A surface element can also be extended slightly at their boundaries to become an embedded $C^{1}$-manifold, except possibly at some finitely many boundary points (See Figure 5 (a)).

We will call a compact set in $\mathbb{R}^{3}$ pseudonormal, if its MA and MAT satisfy the above conditions. In fact, the finiteness of the number of elements, which is connected to the compactness of MA and MAT, is quite important and subtle in proving our results. On the 


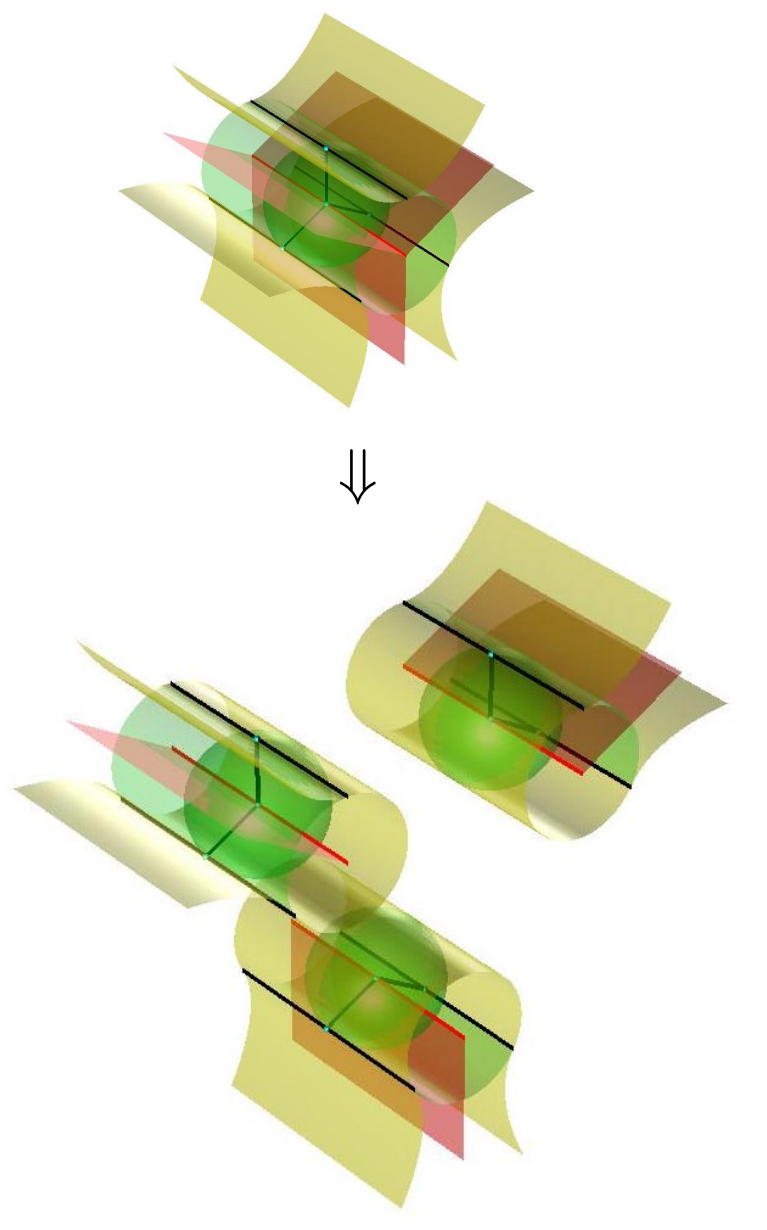

Figure 6: Domain decomposition for 3D Domain: Corresponding to their MAT decomposition, 3D domains can also be decomposed into simpler ones.

domain side, the notion corresponding to MA and MAT decomposition into patches, would be the domain decomposition. See Figure 6 for an example of domain decomposition for 3D domain.

Now, following the definition in $2 \mathrm{D}$, we call a compact set $\Omega$ in $\mathbb{R}^{3}$ normal, if its boundary $\partial \Omega$ consists of finitely many (mutually disjoint) piecewise real-analytic compact 2-manifolds (without boundary). Note that we can easily cook up non-normal examples of 3D domains (analogous to the ones in [4]) whose MAs and MATs inevitably have infinitely many patches, thus not being pseudonormal. But in view of Proposition 1, we can justifiably conjecture the following:

CONJECTURE 1. If a compact set in $\mathbb{R}^{3}$ is normal, then it is pseudonormal.

For the rest of our analysis, we will confine ourselves to pseudonormal domains. Let $\Omega$ be a pseudonormal domain. Let $P=(p, r)$ be a point in $\operatorname{MAT}(\Omega)$, which is not on the boundaries of elements of $\operatorname{MAT}(\Omega)$. There are two types of such generic points in $\operatorname{MAT}(\Omega)$. One is on a surface element, and the other is on a line element. See Figure 7. If $P$ is on a surface element, then the maximal ball $B_{r}(p)$ has exactly two contact points with the boundary $\partial \Omega$. In this case, we define an angle $0<\theta(p) \leq \frac{\pi}{2}$ by the angle $\frac{1}{2} \angle q_{1} p q_{2}$, where $q_{1}, q_{2} \in \partial \Omega \cap \partial B_{r}(p)$ are the contact points. If
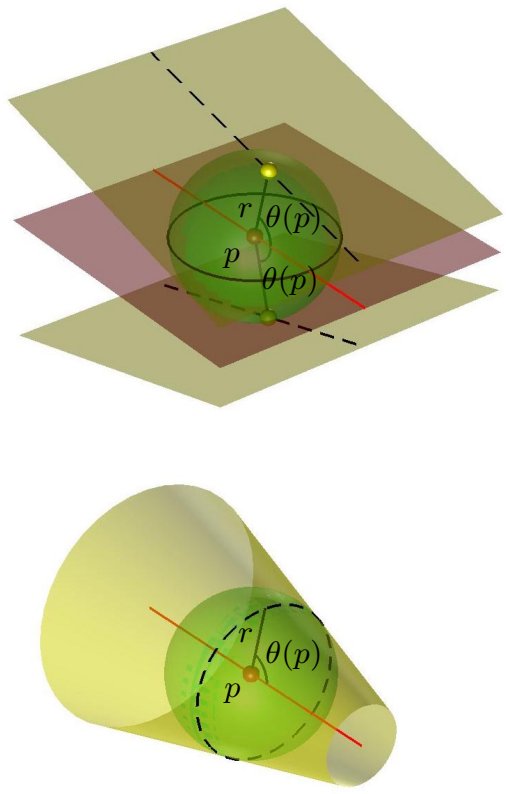

Figure 7: Two kinds of generic points in $\operatorname{MAT}(\Omega)$ : (Up) Generic point in a surface element. The maximal ball has exactly two contact points with the domain boundary. (Below) Generic point in a line element. The contact points form a circle, which, together with the MA point, makes a cone.

$P$ is on a line element, then the contact points form a circle. In this case, we define $\theta(p)$ to be the inner angle of the cone generated by $p$ and the circle of contact points. For both cases, we define an angle $0 \leq \alpha(p)<\frac{\pi}{4}$ by

$$
\tan \alpha(p)=\cos \theta(p) .
$$

Analogously to $2 \mathrm{D}$, we define (except when $\Omega$ is a spherical ball)

$$
\begin{aligned}
\theta_{\Omega} & =\inf \{\theta(p): p \in G(\Omega)\}, \\
\alpha_{\Omega} & =\sup \{\alpha(p): p \in G(\Omega)\},
\end{aligned}
$$

where $G(\Omega)$ denotes the set of all such generic points in $\mathbf{M A}(\Omega)$. Then, from (2), we have

$$
\cos \theta_{\Omega}=\tan \alpha_{\Omega} .
$$

Note that $0 \leq \theta_{\Omega} \leq \frac{\pi}{2}$ and $0 \leq \alpha_{\Omega} \leq \frac{\pi}{4}$. When $\Omega$ is a spherical ball, then we define $\theta_{\Omega}=\frac{\pi}{2}$ and $\alpha_{\Omega}=0$. We call a pseudonormal domain weakly injective, if $\theta_{\Omega}>0$, or equivalently, $\alpha_{\Omega}<\frac{\pi}{4}$.

Like the 2D case, we call an end point of MA (or MAT) a 1prong point. But in $3 \mathrm{D}$, we have more types of 1-prong points than in 2D, some of which are depicted in Figure 8. Here, the center of a spherical ball corresponds to Type (a1). It is easy to see that, if a pseudonormal domain is weakly injective, then its MA cannot have 1-prong points of Type (c1) and (c2) in Figure 8. In general, there occurs a principal curvature maximum at the boundary point corresponding to an MA point of Type (c), although the converse is not always true. This is a useful criterion when determining whether a given shape is weakly injective or not.

Note that a weakly injective 3D domain may have sharp corners or edges (Type(b1) and (b2) in Figure 8). See Figure 9 for an example of weakly injective 3D domain, and another which is not weakly injective. 


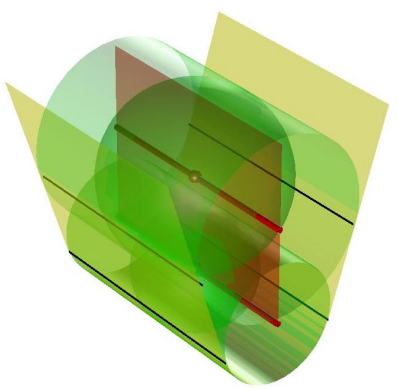

(a2)

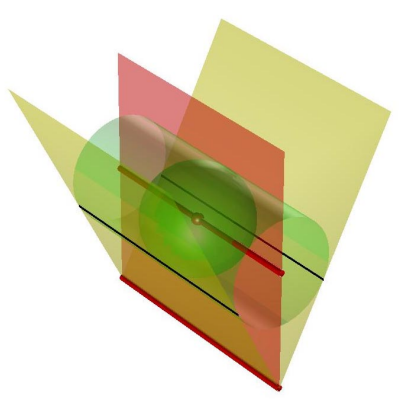

(b2)

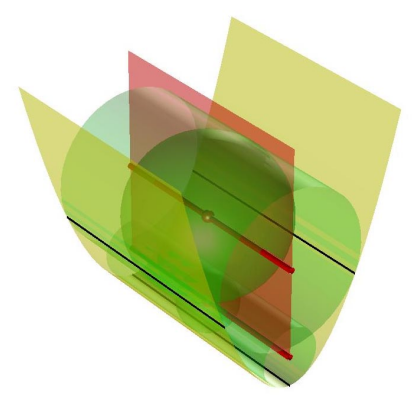

(c2)

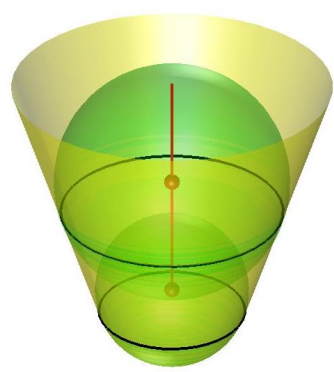

(a1)

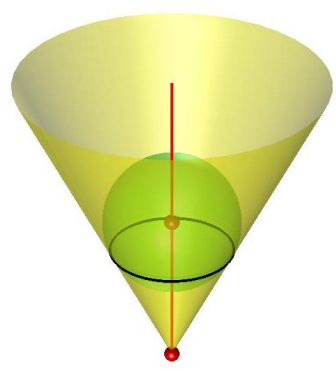

(b1)

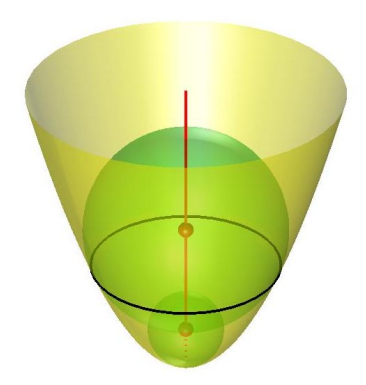

(c1)
Figure 8: Types of 1-prong points in 3D MAT: In addition to these, there are other types which are more 3D specific.

\section{DISTANCES}

\subsection{Hausdorff Distances}

We will use the Hausdorff distance in our analysis, since it is a most natural device to measure the difference between shapes. In fact, we will use three different types of Hausdorff distances: the one-sided, the two-sided and the hyperbolic, where the last one has recently been introduced specifically for MAT. Let $A$ and $B$ be two (compact) sets in a Euclidean space of arbitrary dimension, and let $d(\cdot, \cdot)$ be the usual Euclidean distance.

\section{One-sided Hausdorff distance: $\mathcal{H}(A \mid B)$}

The one-sided Hausdorff distance of $A$ with respect to $B$, denoted by $\mathcal{H}(A \mid B)$, is defined by

$$
\mathcal{H}(A \mid B)=\max _{p \in A} d(p, B) .
$$

Note that $\mathcal{H}(A \mid B)<\epsilon$, if and only if $A$ is contained in the $\epsilon$ -
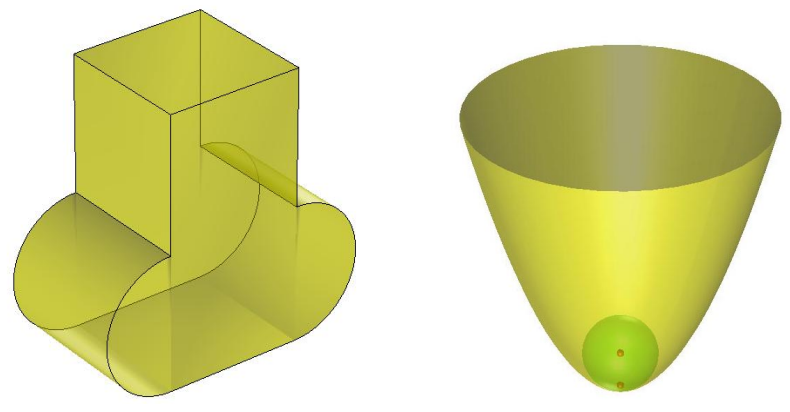

Figure 9: Examples of 3D domains: (left) weakly injective and (right) not weakly injective.

neighborhood of $B$. So the one-sided Hausdorff distance measures how approximately a set is contained in another set, and hence our results on the one-sided stability of MAT (Theorem 1) will be concerned with bounding this distance for MAT.

\section{(Two-sided) Hausdorff distance: $\mathcal{H}(A, B)$}

The two-sided Hausdorff distance (or just the Hausdorff distance) between $A$ and $B, \mathcal{H}(A, B)$, is defined by

$$
\mathcal{H}(A, B)=\max \{\mathcal{H}(A \mid B), \mathcal{H}(B \mid A)\}
$$

This is the one usually called the Hausdorff distance in the literature. It has many nice properties including that it is a complete metric on the class of all compact sets in the Euclidean space [11]. Since it is defined by symmetrizing the one-sided Hausdorff distance, it measures how similar two sets are. Later in Section 6, we will obtain a bound (Corollary 1) for the two-sided Hausdorff distance between two different MAT approximations.

Hyperbolic Hausdorff distance: $\mathcal{H}_{h}\left(M_{1} \mid M_{2}\right), \mathcal{H}_{h}\left(M_{1}, M_{2}\right)$

In spite of its intuitive appeal, the Hausdorff distance cannot capture the unstable behaviour of MAT under the boundary perturbation. The hyperbolic Hausdorff distance has recently been introduced in [8], so that MAT becomes stable if the difference between two MATs is measured by this distance (See Proposition 2 below).

Since MAT is the set of pairs of centers (points in the usual Euclidean space) and radii (nonnegative real numbers), the natural space where MAT lives is the product space $\mathbb{R}^{n} \times \mathbb{R}_{\geq 0}$. The hyperbolic Hausdorff distance measures the difference between two sets in this space. It is defined in a similar way to the usual Hausdorff distance, but uses the hyperbolic distance between two points in $\mathbb{R}^{n} \times \mathbb{R}_{\geq 0}$ instead of the usual Euclidean distance. Let $P_{1}=$ $\left(p_{1}, r_{1}\right), P_{2}=\left(p_{2}, r_{2}\right)$ be in $\mathbb{R}^{n} \times \mathbb{R}_{\geq 0}$. Then the hyperbolic distance $d_{h}\left(P_{1} \mid P_{2}\right)$ from $P_{1}$ to $P_{2}$ is defined by

$$
d_{h}\left(P_{1} \mid P_{2}\right)=\max \left\{0, d\left(p_{1}, p_{2}\right)-\left(r_{2}-r_{1}\right)\right\} .
$$

Note that this is not a real distance, since it is not symmetric (i.e., $d_{h}\left(P_{1} \mid P_{2}\right) \neq d_{h}\left(P_{2} \mid P_{1}\right)$ in general). But, analogously to the usual Euclidean distance, the hyperbolic distance has some nice properties like the following triangular inequality [8]:

$$
d_{h}\left(P_{1} \mid P_{3}\right) \leq d_{h}\left(P_{1} \mid P_{2}\right)+d_{h}\left(P_{2} \mid P_{3}\right),
$$

for any $P_{1}, P_{2}, P_{3} \in \mathbb{R}^{n} \times \mathbb{R}_{\geq 0}$.

Now let $M_{1}, M_{2}$ be compact sets in $\mathbb{R}^{n} \times \mathbb{R}_{\geq 0}$. Then the onesided hyperbolic Hausdorf distance $\mathcal{H}_{h}\left(M_{1} \mid M_{2}\right)$ of $M_{1}$ with re- 
spect to $M_{2}$ is defined by

$$
\mathcal{H}_{h}\left(M_{1} \mid M_{2}\right)=\max _{P_{1} \in M_{1}}\left\{\min _{P_{2} \in M_{2}} d_{h}\left(P_{1} \mid P_{2}\right)\right\},
$$

and the (two-sided) hyperbolic Hausdorff distance between $M_{1}$ and $M_{2}$ is defined by

$$
\mathcal{H}_{h}\left(M_{1}, M_{2}\right)=\max \left\{\mathcal{H}_{h}\left(M_{1} \mid M_{2}\right), \mathcal{H}_{h}\left(M_{2} \mid M_{1}\right)\right\} .
$$

The following fact says that the hyperbolic Hausdorff distance between two MATs is almost the same with the Hausdorff distance between the corresponding original shapes. This implies that the hyperbolic Hausdorff distance is a most natural device to measure the difference between MATs.

Proposition 2. [8]

For any compact sets $\Omega_{1}, \Omega_{2} \subset \mathbb{R}^{n}(n=1,2, \cdots)$ such that $\operatorname{MAT}\left(\Omega_{1}\right), \operatorname{MAT}\left(\Omega_{2}\right)$ are compact, we have

$$
\begin{gathered}
\max \left\{\mathcal{H}\left(\Omega_{1}, \Omega_{2}\right), \mathcal{H}\left(\partial \Omega_{1}, \partial \Omega_{2}\right)\right\} \leq \\
\mathcal{H}_{h}\left(\mathbf{M A T}\left(\Omega_{1}\right), \operatorname{MAT}\left(\Omega_{2}\right)\right), \\
\mathcal{H}_{h}\left(\mathbf{M A T}\left(\Omega_{1}\right), \boldsymbol{M A T}\left(\Omega_{2}\right)\right) \leq \\
3 \cdot \max \left\{\mathcal{H}\left(\Omega_{1}, \Omega_{2}\right), \mathcal{H}\left(\partial \Omega_{1}, \partial \Omega_{2}\right)\right\} .
\end{gathered}
$$

In this paper, the second inequality of Proposition 2 will be used in a crucial way to show the main results regarding the one-sided stability of MATs of weakly injective 3D domains.

\subsection{Distance between Two Points}

Now we will explore some relations between the hyperbolic distance and the Euclidean distance between two MAT points (hence, two points in $\mathbb{R}^{n} \times \mathbb{R}_{\geq 0}$ ), which is important for understanding the further analysis. For concreteness, we will restrict to $\mathbb{R}^{3} \times \mathbb{R}_{\geq 0}$, though the relations hold in every dimension. Let $P_{i}=\left(p_{i}, r_{i}\right)$, $i=1,2$, be two different points in $\mathbb{R}^{3} \times \mathbb{R}_{\geq 0}$. We will denote by $-\frac{\pi}{2} \leq \alpha\left(P_{1}, P_{2}\right) \leq \frac{\pi}{2}$, the angle from $\mathbb{R}^{3} \times\{0\}$ (the space for centers) to $\overrightarrow{P_{1} P_{2}}$ in $\mathbb{R}^{3} \times \mathbb{R}_{\geq 0}$. More specifically, $\alpha\left(P_{1}, P_{2}\right)$ is given by

$$
\sin \alpha\left(P_{1}, P_{2}\right)=\overrightarrow{e_{r}} \cdot \frac{\overrightarrow{P_{1} P_{2}}}{d\left(P_{1}, P_{2}\right)}
$$

where $\overrightarrow{e_{r}}=((0,0,0), 1)$. Here $d\left(P_{1}, P_{2}\right)$ denotes the usual Euclidean distance

$$
d\left(P_{1}, P_{2}\right)=\sqrt{d\left(p_{1}, p_{2}\right)^{2}+\left(r_{1}-r_{2}\right)^{2}},
$$

with $P_{1}, P_{2}$ regarded as points in $\mathbb{R}^{4} \supset \mathbb{R}^{3} \times \mathbb{R}_{\geq 0}$. See Figure 10 for an illustration.

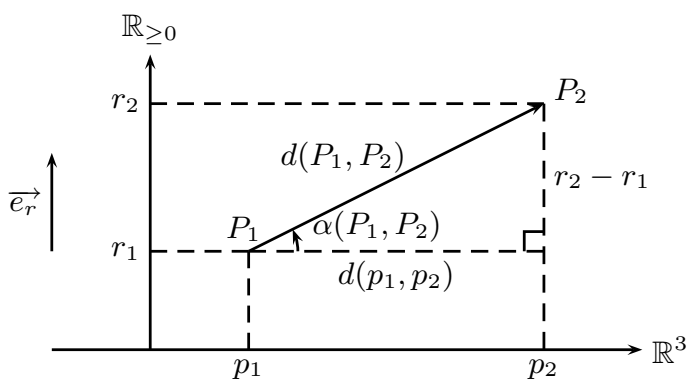

Figure 10: Two points in $\mathbb{R}^{3} \times \mathbb{R}_{\geq 0}$
Suppose $P_{1}, P_{2}$ are two different points in the MAT of a 3D domain $\Omega$. Then we have the following basic observation from the maximality of the two balls $B_{r_{1}}\left(p_{1}\right), B_{r_{2}}\left(p_{2}\right)$ in $\operatorname{MAT}(\Omega)$ :

$$
\left|\alpha\left(P_{1}, P_{2}\right)\right|<\frac{\pi}{4} .
$$

Now from (4), it is clear that

$$
d_{h}\left(P_{1} \mid P_{2}\right)=\left\{\cos \alpha\left(P_{1}, P_{2}\right)-\sin \alpha\left(P_{1}, P_{2}\right)\right\} \cdot d\left(P_{1}, P_{2}\right) .
$$

So we have

$$
\frac{d\left(P_{1}, P_{2}\right)}{d_{h}\left(P_{1} \mid P_{2}\right)}=\frac{1}{\cos \alpha\left(P_{1}, P_{2}\right)-\sin \alpha\left(P_{1}, P_{2}\right)} .
$$

\section{PREVIOUS RESULTS IN 2D}

Recently, there has been a series of results on the one-sided stability of the MAT in 2D. Let us briefly review these results. Chronologically, they were developed in the order of the restrictiveness of the domains dealt with: the injective, the weakly injective, and the general normal. The first result was for injective domains.

Proposition 3. (One-sided Stability for Injective 2D Domain) $[6,7]$

Let $\Omega$ be an injective $2 D$ domain. Then we have

$$
\begin{aligned}
& \mathcal{H}\left(\mathbf{M A}(\Omega) \mid \mathbf{M A}\left(\Omega^{\prime}\right)\right) \\
& \leq \frac{2}{1-\cos \theta_{\Omega}} \cdot \epsilon+o(\epsilon), \\
& \mathcal{H}\left(\mathbf{M A T}(\Omega) \mid \operatorname{MAT}\left(\Omega^{\prime}\right)\right) \\
& \leq \frac{\sqrt{4+\left(3-\cos \theta_{\Omega}\right)^{2}}}{1-\cos \theta_{\Omega}} \cdot \epsilon+o(\epsilon),
\end{aligned}
$$

for every normal $2 D$ domain $\Omega^{\prime}$ such that

$$
\max \left\{\mathcal{H}\left(\Omega, \Omega^{\prime}\right), \mathcal{H}\left(\partial \Omega, \partial \Omega^{\prime}\right)\right\} \leq \epsilon
$$

After the introduction of the hyperbolic Hausdorff distance in [8], this result was extended to the weakly injective case and the general normal case.

PROPOSITION 4. (One-sided Stability for Weakly Injective 2D Domain) [7]

Let $\Omega$ be a weakly injective $2 D$ domain. Then we have

$$
\begin{aligned}
\mathcal{H}\left(\mathbf{M A T}(\Omega) \mid \mathbf{M A T}\left(\Omega^{\prime}\right)\right) & \leq g\left(\theta_{\Omega}\right) \cdot \epsilon+o(\epsilon), \\
\mathcal{H}\left(\mathbf{M A}(\Omega) \mid \mathbf{M A}\left(\Omega^{\prime}\right)\right) \leq & \leq\left(\theta_{\Omega}\right) \cdot \epsilon+o(\epsilon),
\end{aligned}
$$

for every normal $2 D$ domain $\Omega^{\prime}$ such that

$$
\max \left\{\mathcal{H}\left(\Omega, \Omega^{\prime}\right), \mathcal{H}\left(\partial \Omega, \partial \Omega^{\prime}\right)\right\} \leq \epsilon .
$$

Here,

$$
g(\theta)=3\left(1+\frac{2 \sqrt{1+\cos ^{2} \theta}}{1-\cos \theta}\right),
$$

for $\theta \in(0, \pi / 2]$. See Figure 11 for the graph of $g$.

The bounds are linear in the weakly injective case. But it turns out that this linearity does not hold in the general case of the normal domains in 2D. 


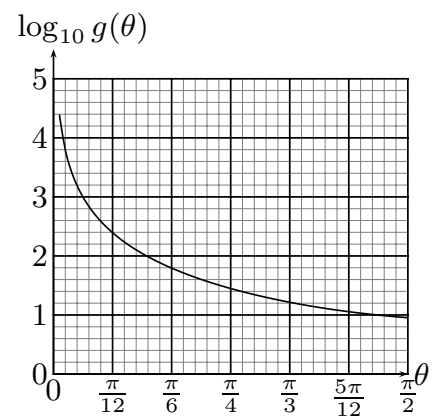

(a)

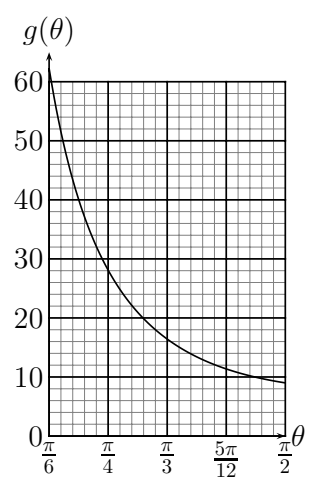

(b)

Figure 11: The graph of the coefficient function $g(\theta)=$ $3\left(1+\frac{2 \sqrt{1+\cos ^{2} \theta}}{1-\cos \theta}\right) ;$ (a) the logarithmic graph of $g$ on the whole interval $\left(0, \frac{\pi}{2}\right]$, (b) the (normal) graph of $g$ on the interval $\left[\frac{\pi}{6}, \frac{\pi}{2}\right]$. [9]

Proposition 5. (One-sided Stability for Normal 2D Domain)

Let $\Omega$ be a normal $2 D$ domain which is not weakly injective. Then we have

$$
\begin{aligned}
& \mathcal{H}\left(\mathbf{M A}(\Omega) \mid \mathbf{M A}\left(\Omega^{\prime}\right)\right) \\
& \leq K_{\Omega} \cdot \epsilon^{\frac{N_{\Omega}-1}{N_{\Omega}+1}}+o\left(\epsilon^{\frac{N_{\Omega}-1}{N_{\Omega}+1}}\right) \\
& \mathcal{H}\left(\mathbf{M A T}(\Omega) \mid \operatorname{MAT}\left(\Omega^{\prime}\right)\right) \\
& \leq K_{\Omega} \cdot \epsilon^{\frac{N_{\Omega}-1}{N_{\Omega}+1}}+o\left(\epsilon^{\frac{N_{\Omega}-1}{N_{\Omega}+1}}\right),
\end{aligned}
$$

for every normal $2 D$ domain $\Omega^{\prime}$ such that

$$
\max \left\{\mathcal{H}\left(\Omega, \Omega^{\prime}\right), \mathcal{H}\left(\partial \Omega, \partial \Omega^{\prime}\right)\right\} \leq \epsilon
$$

Here the constant $N_{\Omega}$ is the degree of contact of maximal balls of Type (c) in Figure 3 with the domain boundary. So $N_{\Omega}$ can take an integer value greater or equal to 2 , and hence we have bounds like $\sqrt[3]{\epsilon}, \sqrt{\epsilon}, \epsilon^{\frac{3}{5}}, \cdots$. In the worst case, we have $\sqrt[3]{\epsilon}$, and, when the boundary curves are quadratic splines, we have $\sqrt{\epsilon}$. The constant $K_{\Omega}$ depends on the maximal curvature of the boundary near 1-prong points of Type (c) in Figure 3. This implies that, in contrast to the constant $\theta_{\Omega}$ in the weakly injective case, we can compute $K_{\Omega}$ directly from the domain boundary.

\section{RESULT IN 3D}

We now show that, the one-sided Hausdorff distance of the MAT (and the MA) of a weakly injective 3D domain is bounded linearly by the magnitude of the boundary perturbation. For these results, we will need the following key lemmas:

LEMMA 1. Let $\Omega$ be a weakly injective $3 D$ domain which is not a spherical ball. Then we have

$$
\begin{array}{r}
\sup \left\{\frac{d\left(P_{1}, P_{2}\right)}{d_{h}\left(P_{1} \mid P_{2}\right)}: P_{1} \neq P_{2} \in \mathbf{M A T}(\Omega), d_{h}\left(P_{1} \mid P_{2}\right) \leq \epsilon\right\} \\
\leq \frac{\sqrt{1+\cos ^{2} \theta_{\Omega}}}{1-\cos \theta_{\Omega}}+o(1)
\end{array}
$$

See Appendix A for the proof of Lemma 1.

Lemma 2. Let $P_{1}, P_{2}, P_{3}$ be in $\mathbb{R}^{3} \times \mathbb{R}_{\geq 0}$. Suppose $d_{h}\left(P_{1} \mid P_{2}\right)$ $\leq \epsilon$ and $d_{h}\left(P_{2} \mid P_{3}\right) \leq \epsilon$ for some $\epsilon \geq 0$. Then we have

$$
d\left(P_{1}, P_{2}\right) \leq d\left(P_{1}, P_{3}\right)+\epsilon .
$$

PROOF. See the proof of Lemma 2 in [7], which can obviously be generalized to arbitrary dimension.

THEOREM 1. (One-sided Stability for Weakly Injective 3D Domain)

Let $\Omega$ be a weakly injective $3 D$ domain. Then we have

$$
\begin{aligned}
\mathcal{H}\left(\mathbf{M A T}(\Omega) \mid \operatorname{MAT}\left(\Omega^{\prime}\right)\right) & \leq g\left(\theta_{\Omega}\right) \cdot \epsilon+o(\epsilon), \\
\mathcal{H}\left(\mathbf{M A}(\Omega) \mid \operatorname{MA}\left(\Omega^{\prime}\right)\right) & \leq g\left(\theta_{\Omega}\right) \cdot \epsilon+o(\epsilon),
\end{aligned}
$$

for every pseudonormal $3 D$ domain $\Omega^{\prime}$ such that $\max \left\{\mathcal{H}\left(\Omega, \Omega^{\prime}\right)\right.$, $\left.\mathcal{H}\left(\partial \Omega, \partial \Omega^{\prime}\right)\right\} \leq \epsilon$.

Once we have Lemmas 1 and 2, the proof of the above theorem is similar to that of the weakly injective 2D case [7]. But for the sake of completeness, we give the proof below. In the proof, note the essential role of the hyperbolic Hausdorff distance (Proposition 2).

Proof of TheOrem 1. First, it is easy to see that $\mathcal{H}\left(\mathbf{M A}(\Omega) \mid \mathbf{M A}\left(\Omega^{\prime}\right)\right) \leq \mathcal{H}\left(\mathbf{M A T}(\Omega) \mid \mathbf{M A T}\left(\Omega^{\prime}\right)\right)$. So we only need to bound the $\mathcal{H}\left(\operatorname{MAT}(\Omega) \mid \operatorname{MAT}\left(\Omega^{\prime}\right)\right)$. Assume $\Omega$ is not a spherical ball. Let $P \in \operatorname{MAT}(\Omega)$. By Proposition 2, we have $\mathcal{H}_{h}\left(\operatorname{MAT}(\Omega), \operatorname{MAT}\left(\Omega^{\prime}\right)\right) \leq 3 \epsilon$. So by (6) and (7), there exists $P^{\prime} \in \operatorname{MAT}\left(\Omega^{\prime}\right)$ such that $d_{h}\left(P \mid P^{\prime}\right) \leq 3 \epsilon$, and, again, there exists $P^{\prime \prime} \in \operatorname{MAT}(\Omega)$ such that $d_{h}\left(P^{\prime} \mid P^{\prime \prime}\right) \leq 3 \epsilon$. So $d_{h}\left(P \mid P^{\prime \prime}\right) \leq 6 \epsilon$ by (5). From Lemma 1 , we have $d\left(P, P^{\prime \prime}\right) \leq \frac{\sqrt{1+\cos ^{2} \theta_{\Omega}}}{1-\cos \theta_{\Omega}} \cdot 6 \epsilon+$ $o(\epsilon)$. So, by Lemma $2, d\left(P, P^{\prime}\right) \leq 3 \epsilon+\frac{\sqrt{1+\cos ^{2} \theta_{\Omega}}}{1-\cos \theta_{\Omega}} \cdot 6 \epsilon+o(\epsilon)=$ $g\left(\theta_{\Omega}\right) \cdot \epsilon+o(\epsilon)$. Since $P$ is taken arbitrarily in $\operatorname{MAT}(\Omega)$, this implies the desired result.

For the case when $\Omega$ is a spherical ball, see the argument in Example 5.

We mention that we can in fact have a globally linear bound; For any weakly injective $3 \mathrm{D}$ domain, we can find a constant $k_{\Omega}<\infty$ such that

$$
\begin{aligned}
\mathcal{H}\left(\operatorname{MAT}(\Omega) \mid \operatorname{MAT}\left(\Omega^{\prime}\right)\right) & \leq k_{\Omega} \cdot \epsilon, \\
\mathcal{H}\left(\mathbf{M A}(\Omega) \mid \mathbf{M A}\left(\Omega^{\prime}\right)\right) & \leq k_{\Omega} \cdot \epsilon,
\end{aligned}
$$

for every $\epsilon \geq 0$, and for every pseudonormal 3D domain $\Omega^{\prime}$ such that $\max \left\{\mathcal{H}\left(\Omega, \Omega^{\prime}\right), \mathcal{H}\left(\partial \Omega, \partial \Omega^{\prime}\right)\right\} \leq \epsilon$.

In the $2 \mathrm{D}$ result [7], it was shown that the bound corresponding to that in Theorem 1 is tight up to constant. This is also true in $3 \mathrm{D}$, which can be seen from the following example.

EXAMPLE 1. Let $\Omega$ be a weakly injective $3 D$ domain with a sharp corner $p_{1}$ depicted as in Figure 12. Let $\Omega^{\prime}$ be the domain obtained by smoothing $\Omega$ with a sphere near $p_{1}$ so that $\mathbf{M A}(\Omega)=$ $\overline{p_{2} p_{3}}$. Let $P_{i}=\left(p_{i}, r_{i}\right)$ be the corresponding points in $\operatorname{MAT}(\Omega)$ for $i=1,2,3$. Note that

$$
\mathcal{H}\left(\Omega, \Omega^{\prime}\right)=\mathcal{H}\left(\partial \Omega, \partial \Omega^{\prime}\right)=\epsilon,
$$

and

$$
\begin{gathered}
\mathcal{H}\left(\mathbf{M A}(\Omega) \mid \mathbf{M A}\left(\Omega^{\prime}\right)\right)=d\left(p_{1}, p_{2}\right)=\frac{1}{1-\cos \theta_{\Omega}} \cdot \epsilon, \\
\mathcal{H}\left(\mathbf{M A T}(\Omega) \mid \mathbf{M A T}\left(\Omega^{\prime}\right)\right)=d\left(P_{1}, P_{2}\right)=\frac{\sqrt{1+\cos ^{2} \theta_{\Omega}}}{1-\cos \theta_{\Omega}} \cdot \epsilon .
\end{gathered}
$$




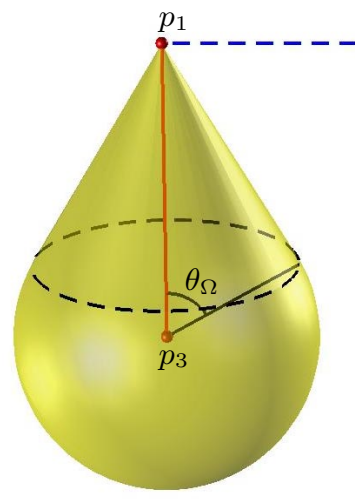

$\Omega$

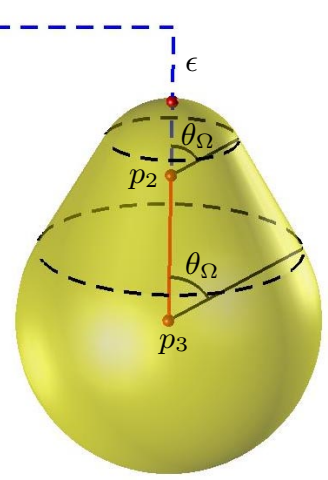

$\Omega^{\prime}$
Figure 12: Example showing the tightness of the bound in Theorem 1.

This example shows that the factor $\frac{1}{1-\cos \theta_{\Omega}}$ in $g\left(\theta_{\Omega}\right)$, which blows up as $\theta_{\Omega} \rightarrow 0$, is indeed unavoidable. In fact, it can be shown that the class of weakly injective 3D domains is the largest possible class for which we have linear bound for the one-sided Hausdorff distance of MAT (and MA) with respect to the boundary perturbation.

Now we calculate explicitly the constants $\theta_{\Omega}$ and $g\left(\theta_{\Omega}\right)$ for a few simple but illustrative examples.

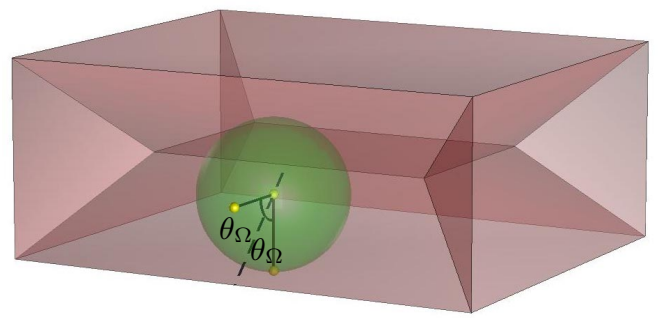

Figure 13: Hexahedron: $\theta_{\Omega}=\frac{\pi}{4}, g\left(\theta_{\Omega}\right)=28.089243 \ldots$

EXAMPLE 2. When $\Omega$ is a hexahedron (Figure 13) or a cylinder (Figure 14), it is easy to see that $\theta_{\Omega}=\frac{\pi}{4}$. So we have $g\left(\theta_{\Omega}\right)=$ $3(1+2 \sqrt{3}(1+\sqrt{2}))=28.089243 \ldots$

EXAMPLE 3. Let $\Omega$ be a regular tetrahedron shown in Figure 15. Here we have $\cos \theta_{\Omega}=\frac{1}{\sqrt{3}}$, and so $\theta_{\Omega}=54.73561 \ldots \circ$. Hence we have $g\left(\theta_{\Omega}\right)=9+6 \sqrt{3}=19.3923 \ldots$.

EXAMPLE 4. Let $\Omega$ be a torus shown in Figure 15. Here we have $\theta_{\Omega}=\frac{\pi}{2}$. Hence we have $g\left(\theta_{\Omega}\right)=9$.

EXAMPLE 5. Let $\Omega$ be the unit spherical ball centered at the origin O. (See Figure 17 (left).) For $\lambda>0$, let $\Omega_{\lambda}$ be a domain such that

$$
\operatorname{MAT}\left(\Omega_{\lambda}\right)=\{((x, 0,0), 1) \mid 0 \leq x \leq \lambda\} .
$$

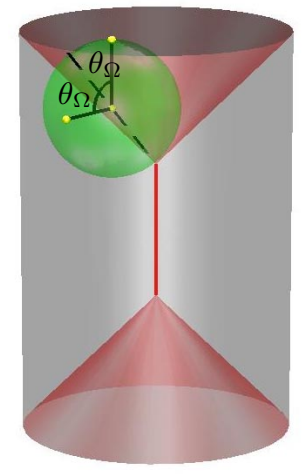

Figure 14: Cylinder: $\theta_{\Omega}=\frac{\pi}{4}, g\left(\theta_{\Omega}\right)=28.089243 \ldots$

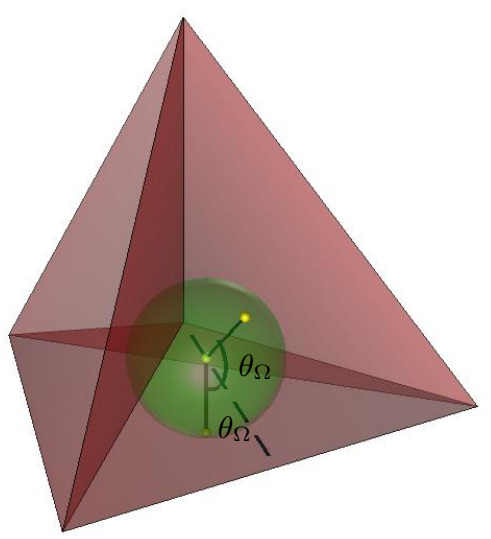

Figure 15: Regular tetrahedron: $\theta_{\Omega}=54.73561 \ldots{ }^{\circ}, g\left(\theta_{\Omega}\right)=$ $19.3923 \ldots$

See Figure 17 (right). Note that, for every $\lambda>0, \Omega_{\lambda}$ is weakly injective, $\theta_{\Omega_{\lambda}}=\frac{\pi}{2}$, and

$$
\max \left\{\mathcal{H}\left(\Omega, \Omega_{\lambda}\right), \mathcal{H}\left(\partial \Omega, \partial \Omega_{\lambda}\right)\right\}=\lambda .
$$

Suppose $\Omega^{\prime}$ is a pseudonormal domain such that

$$
\max \left\{\mathcal{H}\left(\Omega, \Omega^{\prime}\right), \mathcal{H}\left(\partial \Omega, \partial \Omega^{\prime}\right)\right\} \leq \epsilon
$$

Then by the triangular inequality for the Hausdorff distance, it is easy to see that

$$
\max \left\{\mathcal{H}\left(\Omega_{\lambda}, \Omega^{\prime}\right), \mathcal{H}\left(\partial \Omega_{\lambda}, \partial \Omega^{\prime}\right)\right\} \leq \epsilon+\lambda .
$$

By Theorem 1 for non-spherical cases, we have

$$
\begin{aligned}
\mathcal{H}\left(\mathbf{M A T}\left(\Omega_{\lambda}\right) \mid \operatorname{MAT}\left(\Omega^{\prime}\right)\right) & \leq g\left(\frac{\pi}{2}\right) \cdot(\epsilon+\lambda)+o(\epsilon+\lambda), \\
\mathcal{H}\left(\mathbf{M A}\left(\Omega_{\lambda}\right) \mid \mathbf{M A}\left(\Omega^{\prime}\right)\right) & \leq g\left(\frac{\pi}{2}\right) \cdot(\epsilon+\lambda)+o(\epsilon+\lambda) .
\end{aligned}
$$

Since $\operatorname{MAT}(\Omega) \subset \operatorname{MAT}\left(\Omega_{\lambda}\right)$ (and $\mathbf{M A}(\Omega) \subset \mathbf{M A}\left(\Omega_{\lambda}\right)$ ) for every $\lambda>0$, we have

$$
\begin{aligned}
\mathcal{H}\left(\mathbf{M A T}(\Omega) \mid \operatorname{MAT}\left(\Omega^{\prime}\right)\right) & \leq \mathcal{H}\left(\mathbf{M A T}\left(\Omega_{\lambda}\right) \mid \mathbf{M A T}\left(\Omega^{\prime}\right)\right) \\
\mathcal{H}\left(\mathbf{M A}(\Omega) \mid \mathbf{M A}\left(\Omega^{\prime}\right)\right) & \leq \mathcal{H}\left(\mathbf{M A}\left(\Omega_{\lambda}\right) \mid \mathbf{M A}\left(\Omega^{\prime}\right)\right)
\end{aligned}
$$




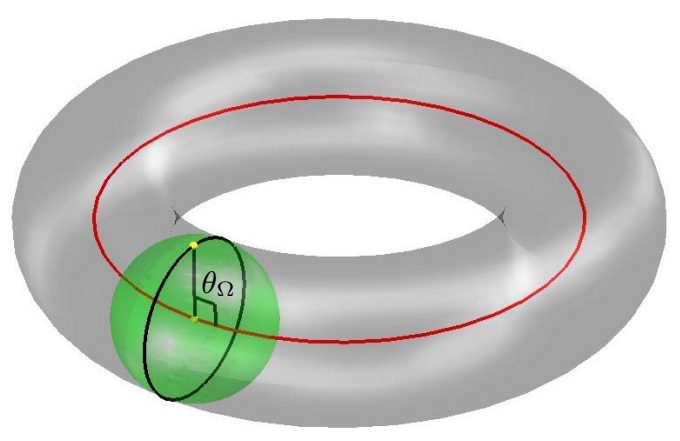

Figure 16: Torus: $\theta_{\Omega}=\frac{\pi}{2}, g\left(\theta_{\Omega}\right)=9$.

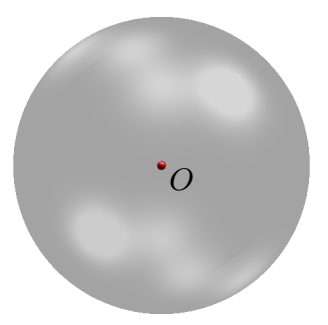

$\Omega$

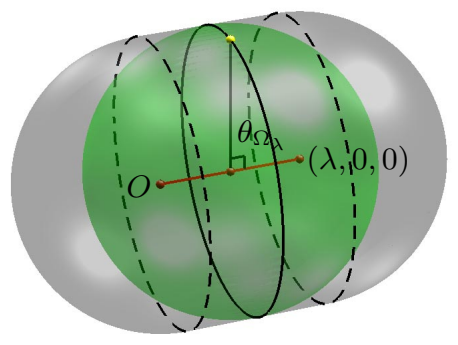

$\Omega_{\lambda}$
Figure 17: Spherical ball and $\Omega_{\lambda}: \theta_{\Omega}=\theta_{\Omega_{\lambda}}=\frac{\pi}{2}, g\left(\theta_{\Omega}\right)=$ $g\left(\theta_{\Omega_{\lambda}}\right)=9$. The spherical ball $\Omega$ can be regarded as the limit of $\Omega_{\lambda}$ as $\lambda \rightarrow 0$.

for every $\lambda>0$. Thus,

$$
\begin{aligned}
\mathcal{H}\left(\mathbf{M A}(\Omega) \mid \mathbf{M A}\left(\Omega^{\prime}\right)\right) & \leq g\left(\frac{\pi}{2}\right) \cdot \epsilon+o(\epsilon), \\
\mathcal{H}\left(\mathbf{M A T}(\Omega) \mid \operatorname{MAT}\left(\Omega^{\prime}\right)\right) & \leq g\left(\frac{\pi}{2}\right) \cdot \epsilon+o(\epsilon),
\end{aligned}
$$

which completes the proof of Theorem 1 for the spherical cases.

EXAMPLE 6. For $n=2,3, \cdots$, let $\Omega_{n}$ be the polyhedral domain as depicted in Figure 18 (top left), which approximates the unit spherical ball centered at the origin as $n \rightarrow \infty$. The vertex points of $\partial \Omega_{n}$ are given by

$$
\left(\cos i \alpha_{n} \cos j \alpha_{n}, \cos i \alpha_{n} \sin j \alpha_{n}, \sin i \alpha_{n}\right),
$$

for $i=-n,-(n-1), \cdots, n$ and $j=1,2, \cdots, 4 n$, where $\alpha_{n}=$ $\frac{\pi}{2 n}$. The MAT of $\Omega_{n}$ has a complex shape consisting of many polygons joining the edges of $\partial \Omega_{n}$ and points (or line segments) on the $z$-axis near the origin. See Figure 18 (top right). By an elementary geometric analysis, it can be shown that

$$
\cos 2 \theta_{\Omega_{n}}=\frac{-\cos ^{2} \alpha_{n}+4 \cos \alpha_{n}+1}{\cos ^{2} \alpha_{n}+3},
$$

and $\theta_{\Omega_{n}}$ is realized at the MAT points which have foot points on the triangular boundary pieces around the poles $(0,0, \pm 1$ ) (Figure 18 (bottom)). Thus, we can see from (9) that $\theta_{\Omega_{n}} \searrow 0$ as $n \rightarrow \infty$ (i.e., as $\Omega_{n}$ approaches the unit spherical ball closer and closer).

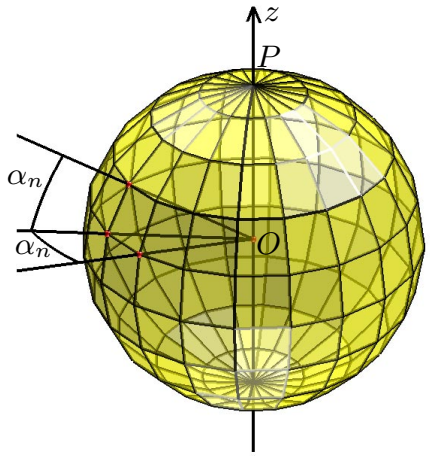

$\Omega_{n}$

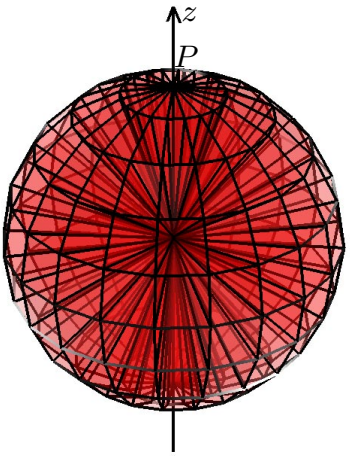

$\operatorname{MA}\left(\Omega_{n}\right)$

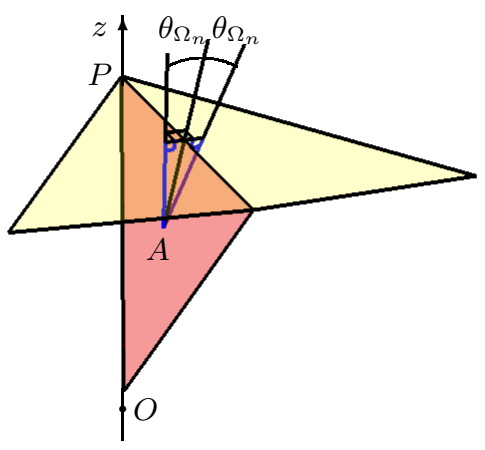

Figure 18: (Top left) the polyhedral domain $\Omega_{n}$ approximating the unit spherical ball centered at the origin, and (Top right) its MA. The vertices of $\partial \Omega_{n}$ are distributed regularly on the unit sphere with the angle $\alpha_{n}=\frac{\pi}{2 n}$. Actually, the origin is slightly outside of the MA pieces (except the ones on the $x y$ plane). (Bottom) The angle $\theta_{\Omega_{n}}$ is realized by a point $A$ on an MA piece between two triangular boundary pieces around the north pole $P$.

\section{CONSEQUENCES}

Now we discuss a few consequences and applications of the onesided stability of 3D MAT. The importance of Theorem 1 lies not only in that it confirms the intuition of the one-sided stability of the MAT, but also in that it provides a quantitative bound of the MAT deviations which is linear in the weakly injective case.

In many situations, a given 3D domain has small noises in its boundary. Yet another situation is that the boundary is given by the popular polygonal mesh which inevitably has numerous small sharp edges. In these cases, it is often more reasonable to compute a simpler approximate MAT, instead of the exact one which contains many unilluminating parts caused by the noise or the local sharp features. A traditional approach to this would be to cut away some parts of the exact MAT according to various existing importance measures [18]. While this approach guarantees that the resulting simpler MAT lies completely inside the exact MAT, one disadvantage is that one still should compute the proliferous exact MAT in advance.

Theorem 1 provides a different strategy: First approximate the given domain with a simpler weakly injective domain and then compute directly the MAT of this simpler domain. Then the resulting simpler MAT is guaranteed to approach to a part of the exact MAT as the Hausdorff distance between the approximating domain and the original one shrinks. Furthermore, we have a quantitative 
bound for the amount of the deviation of the approximating MAT from the exact one.

We can also see that the approximating MAT contains the important global features of the original one.

COROLlaRY 1. (Independence of Approximations)

Let $\Omega$ be a pseudonormal $3 D$ domain, and let $\Omega_{1}$ and $\Omega_{2}$ be two weakly injective $3 D$ domains such that

$$
\begin{gathered}
\max \left\{\mathcal{H}\left(\Omega_{i}, \Omega\right), \mathcal{H}\left(\partial \Omega_{i}, \partial \Omega\right)\right\} \leq \epsilon \\
\text { for } i=1,2 \text {. Let } \theta=\min \left\{\theta_{\Omega_{1}}, \theta_{\Omega_{2}}\right\} . \text { Then we have } \\
\mathcal{H}\left(\mathbf{M A T}\left(\Omega_{1}\right), \operatorname{MAT}\left(\Omega_{2}\right)\right) \leq 2 g(\theta) \cdot \epsilon+o(\epsilon), \\
\mathcal{H}\left(\mathbf{M A}\left(\Omega_{1}\right), \operatorname{MA}\left(\Omega_{2}\right)\right) \leq 2 g(\theta) \cdot \epsilon+o(\epsilon) .
\end{gathered}
$$

PROOF. This follows by applying Theorem 1 symmetrically to $\Omega_{1}$ and $\Omega_{2}$, and from the fact that $\mathcal{H}\left(\Omega_{1}, \Omega_{2}\right) \leq \mathcal{H}\left(\Omega_{1}, \Omega\right)+$ $\mathcal{H}\left(\Omega_{2}, \Omega\right) \leq 2 \epsilon, \mathcal{H}\left(\partial \Omega_{1}, \partial \Omega_{2}\right) \leq \mathcal{H}\left(\partial \Omega_{1}, \partial \Omega\right)+\overline{\mathcal{H}}\left(\partial \Omega_{2}, \partial \Omega\right) \leq$ $2 \epsilon$.

Note that the Hausdorff distances in Corollary 1 are the twosided ones. Corollary 1 tells the following: Suppose we have two different approximating weakly injective domains $\Omega_{1}$ and $\Omega_{2}$ to a given pseudonormal domain $\Omega$. Then the difference between $\operatorname{MAT}\left(\Omega_{1}\right)$ and $\operatorname{MAT}\left(\Omega_{2}\right)$ under the two-sided Hausdorff distance is also bounded linearly. This means that, even if we choose only one approximation, it is guaranteed to contain approximately a common part of the exact MAT which is (approximately) shared by all the other approximations.

As yet another situation, suppose that, instead of approximating only an important part of the MAT, we want to approximate the whole part of the exact MAT of a noiselessly given weakly injective domain, which often appears in CAD applications. For example, this was nicely achieved in $2 \mathrm{D}$ by successively finding the important maximal balls, and interpolating them with splines [5]. In this type of approach, Corollary 1 can also be served for bounding the difference between the interpolated MAT and the exact one.

Finally, we observe from Theorem 1 and Examples 1 and 6 that the angle $\theta_{\Omega}$ is an important quantity reflecting the degree of the "detailed-ness" of a weakly injective domain $\Omega$; Suppose we are approximating a given pseudonormal domain by a weakly injective domain $\Omega$. Then, in general, the value of $\theta_{\Omega}$ gets small as the approximation gets finer. Thus, the angle $\theta_{\Omega}$ can be used as a new indicator of the level-of-detail for 3D domains in the weakly injective class.

\section{REFERENCES}

[1] N. Amenta, S. Choi, and R. K. Kolluri. The power crust. In Proc. Sixth ACM Symp. Solid Modeling and Applications, pages 249-260, Ann Arbor, Michigan, June 2001.

[2] J. August, K. Siddiqi, and S. W. Zucker. Ligature instabilities and the perceptual organization of shape. Computer Vision and Image Understanding, 76(3):231-243, 1999.

[3] H. Blum. A transformation for extracting new descriptors of shape. In W. W. Dunn, editor, Proc. Symp. Models for the Perception of Speech and Visual Form, pages 362-380. MIT Press, Cambridge, MA, 1967.

[4] H. I. Choi, S. W. Choi, and H. P. Moon. Mathematical theory of medial axis transform. Pacific J. Math., 181(1):57-88, 1997.

[5] H. I. Choi, S. W. Choi, H. P. Moon, and N.-S. Wee. New algorithm for medial axis transform of plane domain. Graphical Models and Image Processing, 59(6):464-483, 1997.
[6] S. W. Choi and S.-W. Lee. Stability analysis of medial axis transform under relative Hausdorff distance. In Proc. 15th International Conference on Pattern Recognition, volume 3, pages 139-142, Barcelona, Spain, September 2000.

[7] S. W. Choi and H.-P. Seidel. Linear one-sided stability of MAT for weakly injective domain. Journal of Mathematical Imaging and Vision. to appear.

[8] S. W. Choi and H.-P. Seidel. Hyperbolic Hausdorff distance for medial axis transform. Graphical Models, 63(5):369-384, 2001.

[9] S. W. Choi and H.-P. Seidel. One-sided stability of MAT and its applications. In Proc. Vision, Modeling, and Visualization 2001, pages 291-298, Stuttgart, Germany, November 2001.

[10] M. P. Deseilligny, G. Stamon, and C. Y. Suen. Veinerization: A new shape descriptor for flexible skeletonization. IEEE Trans. Pattern Analysis and Machine Intelligence, 20(5):505-521, 1998.

[11] J. Dugundji. Topology. Allyn and Bacon, Inc., 1966.

[12] P. J. Giblin and B. B. Kimia. On the local form and transitions of symmetry sets, medial axes, and shocks. In Proc. 7th International Conference on Computer Vision, pages 385-391, Kerkyra, Greece, September 1999.

[13] T. Kulver, J. Keyser, and D. Manocha. Accurate computation of the medial axis of a polyhedron. In Proc. Fifth ACM Symp. Solid Modeling and Applications, pages 179-190, Ann Arbor, Michigan, June 1999.

[14] B. Moayer and K. S. Fu. A syntactic approach of fingerprint pattern recognition. Pattern Recognition, 7:1-23, 1975.

[15] F. Mokhtarian and A. K. Mackworth. A theory of multiscale, curvature-based shape representation for planar curves. IEEE Trans. Pattern Analysis and Machine Intelligence, 14(8):789-805, 1992.

[16] U. Montanari. A method for obtaining skeletons using a quasi-Euclidean distance. J. of the ACM, 15(4):600-624, 1968.

[17] T. V. Nguyen and J. Sklansky. A fast skeleton-finder for coronary arteries. In Proc. 8th International Conference on Pattern Recognition, pages 481-483, Paris, France, 1986.

[18] D. Shaked and A. M. Bruckstein. Pruning medial axes. Computer Vision and Image Understanding, 69(2):156-169, 1998.

[19] E. C. Sherbrooke, N. M. Patrikalakis, and E. Brisson. An alogrithm for the medial axis transform of $3 \mathrm{D}$ polyhedral solids. IEEE Trans. Visualization and Computer Graphics, 2:44-61, 1996.

[20] E. C. Sherbrooke, N. M. Patrikalakis, and F.-E. Wolter. Differential and topological properties of medial axis transform. Graphical Models and Image Processing, 58(6):574-592, 1996.

[21] C. Y. Suen, M. Berthold, and S. Mori. Automatic recognition of handprinted characters. Proceedings of the IEEE, 68(4):469-487, 1980.

[22] Q.-Z. Ye and P. E. Danielsson. Inspection of printed circuit boards by connectivity preserving shrinking. IEEE Trans. Pattern Analysis and Machine Intelligence, 10(5):737-742, 1988. 


\section{APPENDIX}

\section{A. PROOF OF LEMMA 1}

Before we proceed, we mention that the compactness of the MAT, which is connected to the finiteness of the number of elements, is a key ingredient in the proof. Let

$A_{\epsilon}=\sup \left\{\alpha\left(P_{1}, P_{2}\right): P_{1} \neq P_{2} \in \mathbf{M A T}(\Omega), d_{h}\left(P_{1} \mid P_{2}\right) \leq \epsilon\right\}$.

Note that $\left|A_{\epsilon}\right| \leq \frac{\pi}{4}$ for any $\epsilon>0$, since $\left|\alpha\left(P_{1}, P_{2}\right)\right|<\frac{\pi}{4}$ for any $P_{1} \neq P_{2} \in \operatorname{MAT}(\Omega)$. We will show that $\lim \sup _{\epsilon \rightarrow 0} A_{\epsilon} \leq \alpha_{\Omega}$. Then the proof would follow, since

$$
\begin{aligned}
B_{\epsilon} & \leq \frac{1}{\cos \left(\tan \alpha_{\Omega}\right)-\sin \left(\tan \alpha_{\Omega}\right)} \\
& =\frac{\sqrt{1+\cos ^{2} \theta_{\Omega}}}{1-\cos \theta_{\Omega}},
\end{aligned}
$$

by (3) and (8), where

$B_{\epsilon}=\sup \left\{\frac{d\left(P_{1}, P_{2}\right)}{d_{h}\left(P_{1} \mid P_{2}\right)}: P_{1} \neq P_{2} \in \mathbf{M A T}(\Omega), d_{h}\left(P_{1} \mid P_{2}\right) \leq \epsilon\right\}$.

Note that $\alpha_{\Omega}<\frac{\pi}{4}$, since $\Omega$ is weakly injective. Suppose

$$
\limsup _{\epsilon \rightarrow 0} A_{\epsilon}>\alpha_{\Omega} \text {. }
$$

Then there exist sequences $\left\{P_{1, m}=\left(p_{1, m}, r_{1, m}\right)\right\}$ and $\left\{P_{2, m}=\right.$ $\left.\left(p_{2, m}, r_{2, m}\right)\right\}$ in $\mathbf{M A T}(\Omega)$ such that $P_{1, m} \neq P_{2, m}, d_{h}\left(P_{1, m} \mid P_{2, m}\right)$ $\leq 1 / 2^{m}$, and $\lim _{m \rightarrow \infty} \alpha\left(P_{1, m}, P_{2, m}\right)>\alpha_{\Omega}$. Since $\mathbf{M A T}(\Omega)$ is compact, we can assume $d\left(P_{1, m}, P_{1}\right) \rightarrow 0$ and $d\left(P_{2, m}, P_{2}\right) \rightarrow 0$ as $m \rightarrow \infty$ for some $P_{1}, P_{2} \in \mathbf{M A T}(\Omega)$. Since $d_{h}\left(P_{1, m} \mid P_{2, m}\right) \rightarrow$ 0 as $m \rightarrow \infty$, it follows that $P_{1}=P_{2}$, which we denote $P=$ $(p, r)$.

We can assume $r_{1, m}<r_{2, m}$ for every $m$, for, otherwise, we would have $\lim _{m \rightarrow \infty} \alpha_{m}=0$, where $\alpha_{m}=\alpha\left(P_{1, m}, P_{2, m}\right)$ for $m=1,2, \cdots$. For the moment, we also assume $r>0$. Since $\Omega$ is weakly injective, this implies that there exist at least two different contact points $q_{1, m}, q_{2, m} \in \partial \Omega \cap \partial B_{r_{1, m}}\left(p_{1, m}\right)$. Let $\theta_{1, m}=$ $\frac{1}{2} \angle q_{1, m} p_{1, m} q_{2, m}\left(0<\theta_{1, m} \leq \frac{\pi}{2}\right)$. See Figure 19. From the

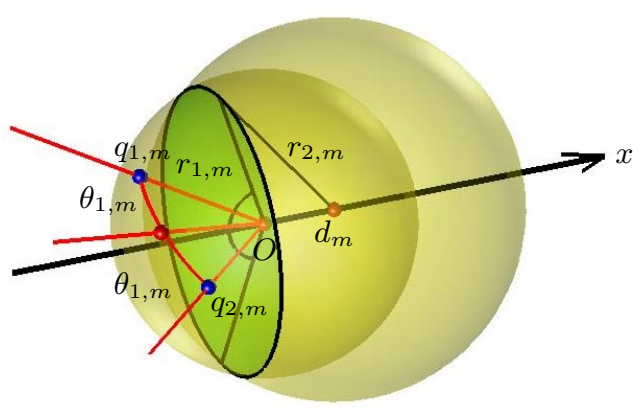

Figure 19: Two spheres $B_{r_{1, m}}\left(p_{1, m}\right)$ and $B_{r_{2, m}}\left(p_{2, m}\right)$ : Since $\Omega$ is weakly injective, there exist two different contact points $q_{1, m}$ and $q_{2, m}$ for each $P_{1, m}$.

definition of $\theta_{\Omega}$, we can see that

$$
\theta_{1, m} \geq \theta_{\Omega},
$$

for every $m$.

Fix $m$. We assume with no loss of generality that $p_{1, m}=(0,0,0)$, $p_{2, m}=\left(d_{m}, 0,0\right)$, where $d_{m}=d\left(p_{1, m}, p_{2, m}\right)>0$. Note that

$$
\tan \alpha_{m}=\frac{r_{2, m}-r_{1, m}}{d_{m}} .
$$

Define $0<\theta_{m} \leq \pi / 2$ as follows: When $d_{m}^{2}+r_{1, m}^{2} \leq r_{2, m}^{2}$, we have the situation shown in Figure 20 (a). Let $q=\left(q_{x}, q_{y}\right)$ $\left(q_{y}>0\right)$ be the point where the two circles intersect. Here $\theta_{m}$ is defined by the angle between $\overline{O\left(q_{x}, q_{y}\right)}$ and the $x$-axis. When $d_{m}^{2}+r_{1, m}^{2}>r_{2, m}^{2}$ (Figure $20(\mathrm{~b})$ ), define $\theta_{m}=\pi / 2$. It is easy to see from Figure 19 that

$$
\theta_{m} \geq \theta_{1, m}
$$

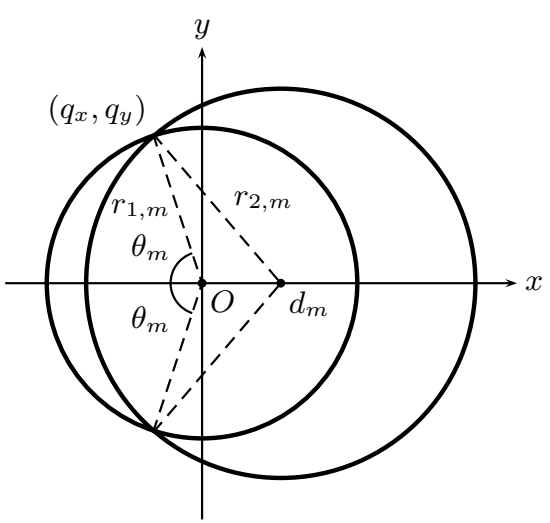

(a)

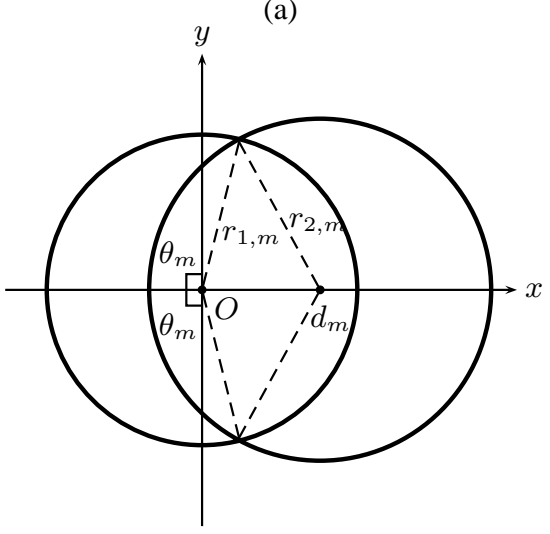

(b)

Figure 20: Two spheres $B_{r_{1, m}}\left(p_{1, m}\right)$ and $B_{r_{2, m}}\left(p_{2, m}\right)$ projected onto the $x y$-plane

Suppose first $d_{m}^{2}+r_{1, m}^{2}<r_{2, m}^{2}$ for infinitely many $m$ 's, in which case, we can assume with no loss of generality that $d_{m}^{2}+$ $r_{1, m}^{2}<r_{2, m}^{2}$ for every $m$. Now we have

$$
\begin{aligned}
q_{x}^{2}+q_{y}^{2} & =r_{1, m}^{2} \\
\left(q_{x}-d_{m}\right)^{2}+q_{y}^{2} & =r_{2, m}^{2}
\end{aligned}
$$

From these, we can easily get

$$
q_{x}=\frac{d_{m}^{2}+r_{1, m}^{2}-r_{2, m}^{2}}{2 d_{m}}
$$

and hence

$$
\cos \theta_{m}=\frac{-d_{m}^{2}-r_{1, m}^{2}+r_{2, m}^{2}}{2 d_{m} r_{1, m}}
$$


From (11) and (13) we have

$$
\begin{aligned}
\cos \theta_{m}-\tan \alpha_{m} & =\frac{-d_{m}^{2}-r_{1, m}^{2}+r_{2, m}^{2}}{2 d_{m} r_{1, m}}-\frac{r_{2, m}-r_{1, m}}{d_{m}} \\
& =\frac{\left(r_{1, m}-r_{2, m}\right)^{2}-d_{m}^{2}}{2 d_{m} r_{1, m}} \\
& =\frac{d_{m}}{2 r_{1, m}}\left(\frac{\left(r_{2, m}-r_{1, m}\right)^{2}}{d_{m}^{2}}-1\right) .
\end{aligned}
$$

So we have

$$
\cos \theta_{m}-\tan \alpha_{m}=-\frac{d_{m}}{2 r_{1, m}}\left(1-\tan ^{2} \alpha_{m}\right) .
$$

From (14), we get

$$
\tan \alpha_{m}-\cos \theta_{m} \rightarrow 0
$$

as $m \rightarrow \infty$. So it follows from (3), (10), and (12) that

$$
\lim _{m \rightarrow \infty} \tan \alpha_{m} \leq \lim _{m \rightarrow \infty} \cos \theta_{1, m} \leq \cos \theta_{\Omega}=\tan \alpha_{\Omega},
$$

and hence

$$
\lim _{m \rightarrow \infty} \alpha_{m} \leq \alpha_{\Omega},
$$

which is a contradiction. So there should be infinitely many $m$ 's such that $d_{m}^{2}+r_{1, m}^{2}>r_{2, m}^{2}$, and we can assume $d_{m}^{2}+r_{1, m}^{2}>r_{2, m}^{2}$ for every $m$. In this case, note that

$$
\begin{aligned}
\tan ^{2} \alpha_{m} & =\frac{\left(r_{2, m}-r_{1, m}\right)^{2}}{d_{m}^{2}} \\
& =\frac{r_{2, m}^{2}-2 r_{2, m} r_{1, m}+r_{1, m}^{2}}{d_{m}^{2}} \\
& =\frac{r_{2, m}^{2}-r_{1, m}^{2}}{d_{m}^{2}}-2 \cdot \frac{r_{2, m}-r_{1, m}}{d_{m}} \cdot \frac{r_{1, m}}{d_{m}} \\
& =\frac{r_{2, m}^{2}-r_{1, m}^{2}}{d_{m}^{2}}-2 \tan \alpha_{m} \cdot \frac{r_{1, m}}{d_{m}} .
\end{aligned}
$$

So we have

$$
\lim _{m \rightarrow \infty} \tan ^{2} \alpha_{m}=\lim _{m \rightarrow \infty} \frac{r_{2, m}^{2}-r_{1, m}^{2}}{d_{m}^{2}}-2 r \cdot \lim _{m \rightarrow \infty} \frac{\tan \alpha_{m}}{d_{m}} .
$$

Since we assumed that $d_{m}^{2}+r_{1, m}^{2}>r_{2, m}^{2}$, we have

$$
0 \leq \lim _{m \rightarrow \infty} \frac{r_{2, m}^{2}-r_{1, m}^{2}}{d_{m}^{2}} \leq 1 .
$$

So from (15), it follows that

$$
\lim _{m \rightarrow \infty} \tan \alpha_{m}=0
$$

since $\lim _{m \rightarrow \infty} d_{m}=0,0 \leq \lim _{m \rightarrow \infty} \tan \alpha_{m} \leq 1$, and $r>0$. So we again have the contradiction that

$$
\lim _{m \rightarrow \infty} \alpha_{m}=0 \leq \alpha_{\Omega} .
$$

From the above arguments, we must have $r=0$. Now let $\Omega^{\prime}$ be the domain obtained by inflating $\Omega$ by small $\delta>0$, i.e.,

$$
\Omega^{\prime}=\Omega+B_{\delta}(O) .
$$

Then is is easy to see that

$$
\operatorname{MAT}\left(\Omega^{\prime}\right)=\operatorname{MAT}(\Omega)+((0,0,0), \delta) .
$$

Obviously, $\Omega^{\prime}$ is a pseudonormal domain, and it is also weakly injective. Moreover, it is also easy to see that $\alpha_{\Omega}^{\prime}=\alpha_{\Omega}$. Let
$P_{i, m}^{\prime}=\left(p_{i, m}^{\prime}, r_{i, m}^{\prime}\right)=\left(p_{i, m}, r_{i, m}+\delta\right), P^{\prime}=\left(p^{\prime}, r^{\prime}\right)=(p, \delta)$, $d_{m}^{\prime}=d\left(p_{1, m}^{\prime}, p_{2, m}^{\prime}\right)$, and $\alpha_{m}^{\prime}=\alpha\left(P_{1, m}^{\prime}, P_{2, m}^{\prime}\right)$ for $i=1,2$ and $m=1,2, \cdots$. Then it is clear that $d_{m}^{\prime}=d_{m}, \alpha_{m}^{\prime}=\alpha_{m}$ for $i=1,2$ and $m=1,2, \cdots$. Now we can apply the same arguments as above to show that $\lim _{m \rightarrow \infty} \alpha_{m} \leq \alpha_{\Omega^{\prime}}=\alpha_{\Omega}$, since $\lim _{m \rightarrow \infty} r_{1, m}^{\prime}=\lim _{m \rightarrow \infty} r_{2, m}^{\prime}=r^{\prime}=\delta>0$. Thus we have a contradiction, and the proof is complete. 